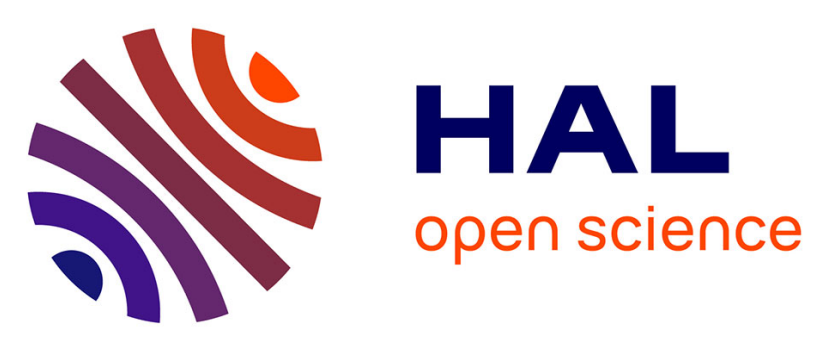

\title{
Numerical analysis of a model for Nickel-Iron alloy electrodeposition on rotating disk electrode
}

Jean Rodolphe Roche, Noureddine Alaa, Aouatif Cheggour, Mohamed Iguernane, Abdessamad Tounsi

\section{- To cite this version:}

Jean Rodolphe Roche, Noureddine Alaa, Aouatif Cheggour, Mohamed Iguernane, Abdessamad Tounsi. Numerical analysis of a model for Nickel-Iron alloy electrodeposition on rotating disk electrode. International Journal of Computer Mathematics, 2008, 85 (10), pp.1531-1549. 10.1080/00207160802033475 . hal-00110540

\section{HAL Id: hal-00110540 \\ https://hal.science/hal-00110540}

Submitted on 30 Oct 2006

HAL is a multi-disciplinary open access archive for the deposit and dissemination of scientific research documents, whether they are published or not. The documents may come from teaching and research institutions in France or abroad, or from public or private research centers.
L'archive ouverte pluridisciplinaire HAL, est destinée au dépôt et à la diffusion de documents scientifiques de niveau recherche, publiés ou non, émanant des établissements d'enseignement et de recherche français ou étrangers, des laboratoires publics ou privés. 


\title{
Numerical analysis of a model for Nickel-Iron alloy electrodeposition on rotating disk electrode*
}

\author{
N. Alaa ${ }^{\dagger}$ A. Cheggour $\ddagger$ M. Iguernane \\ J. R. Rocheqand A. Tounssill
}

\begin{abstract}
To better understand the nickel-iron electrodeposition process, we have developed one-dimensional numerical model. This model addresses dissociation, diffusion, electromigration, convection and deposition of multiple ion species. The reaction mechanism in this model differs in that $\mathrm{Ni}^{2+}$ and $\mathrm{Fe}^{2+}$ are the electroactive species and $\mathrm{NiOH}^{+}$ and $\mathrm{FeOH}{ }^{+}$are not involved whatsover. To take account of the anisotropic behaviour of the solution we introduce a domain decomposition numerical method. Simulations with experimental data shows that our model can predict characteristic features of the nickel-iron system.
\end{abstract}

*this work was supported by the Action Integree program MA/02/33, the "'Agence universitaire de la Francophonie AUF" and the Elie Cartan Institute (IECN) of the Henri Poincaré University

†Faculté des Sciences et Téchniques Gueliz, Département de Mathématiques et Informatique. B.P.549 Marrakech-Maroc, alaa@fstg-marrakech.ac.ma

${ }^{\ddagger}$ Faculté des Sciences et Téchniques Gueliz, Département de Mathématiques et Informatique. B.P.549 Marrakech-Maroc, cheggour@fstg-marrakech.ac.ma

§I.E.C.N., Université Henri Poincaré, B.P 239, 54506 Vandoeuvre lès Nancy, France,iguernan@iecn.u-nancy.fr

ฯI.E.C.N., Université Henri Poincaré, B.P 239, 54506 Vandoeuvre lès Nancy, France,roche@iecn.u-nancy.fr

${ }^{\|}$Faculté des Sciences et Téchniques Errachidia, Département de Chimie. B.P. Errachidia-Maroc, tounssi@fstg-errachidia.ma 


\section{Introduction}

Electrodeposition of alloys based on the iron group of metals is one of the most important recent developments in the field of alloy deposition. Films of NiFe alloy, in particular, have widely been adopted for electronics industry for recording, memory and storage devices [9].

Electrodeposition of $\mathrm{NiFe}$ alloys is anomalous [3]. In the anomalous codeposition, the less noble metal deposits preferentially; consequently its relative content in the alloy deposits is much higher than in the solution.

Since some properties of the film are also strongly related to the alloys content, it is important to understand the effects of various plating operating variables and to be able to control them.

In previous research, most of the proposed models [2], [12] for $\mathrm{NiFe}$ codeposition involve hydrolysis of $\mathrm{Ni}^{2+}$ and $\mathrm{Fe}^{2+}$ ions in solution. In these models, the anomalous codeposition was related to iron hydroxide that precipitated on the electrode surface and inhibited nickel reduction alone. However, it was shown that this hypothesis could not explain some of the experimental data and consequently it was supplanted by another mechanism [7], [6]. According to this newer model, $\mathrm{NiOH}^{+}$and $\mathrm{FeOH}^{+}$formed from the hydrolysis of $\mathrm{Ni}^{2+}$ and $\mathrm{Fe}^{2+}$ are reduced at the cathode. The anomalous codeposition occurs because $\mathrm{FeOH}^{+}$is present near the electrode at higher concentrations than $\mathrm{NiOH}^{+}$.

Despite of the success realized by Gangasing and Talbot [4], Hessami and Tobias [7], Grande and Talbot [6], and Ramasubramanian et al. [14] in the application of this mechanism of codeposition onto a rotating disk electrode some unresolved issues still remain for many reasons [9].

A newer successful model proposed by Matlosz [11] stipulated that the reduction of each metal ion occurs via two-step mechanism with electroabsorption of a monovalent intermediate in the first step and its reduction in the second step. Anomalous codeposition arises from the preferential surface coverage of the adsorbate iron intermediate without having to assume the presence of $\mathrm{NiOH}^{+}$and $\mathrm{FeOH}{ }^{+}$.

In light of this Pritzker et al. [9], [15] proposed transport model for codeposition onto a rotating disk electrode $(R D E)$ without assuming the presence of $\mathrm{NiOH}^{+}$and $\mathrm{FeOH}{ }^{+}$as the electroactive species. As in some of the earlier model developments for $N i F e$ codeposition [7], [6], they consider the system to involve one-dimensional steady-state transport of the various species to a rotating disk electrode $(R D E)$. 
In the present work we conduct a type of modelling similar to that done by Pritzker et al., which is the subject of the section 2. Section 3 is devoted to the numerical simulation of the system. Indeed we propose an iterative scheme and the finite element discretization for resolving the system of equations. Numerical examples are presented in section 4.

\section{The model problem}

We consider the species conservation equation for a species $A_{i}$ as

$$
\frac{\partial w_{i}}{\partial t}=-\operatorname{div}\left(J_{i}\right)+S_{i}
$$

where $w_{i}$ is the concentration of species $A_{i}, S_{i}$ denotes the production rate of $A_{i}$ due to all the homogeneous reactions in which it is involved and $J_{i}$ is its molar transport flux. The electrolytes used for $N i F e$ codeposition always contain electroactive nickel ions as one of their major components. Consequently, migration is included along with diffusion and convection as possible modes of transport for each species. The molar flux $J_{i}$ then becomes

$$
J_{i}=-d_{i} \nabla w_{i}+v w_{i}-m_{i} w_{i} \nabla \Phi
$$

where $d_{i}$ is the diffusion coefficient of species $A_{i}, v$ is the fluid velocity vector, $\Phi$ is the potential and $m_{i}$ is the electrical mobility of species $A_{i}$ (see [16]). The mobility and diffusion coefficient are related through the Einstein equation

$$
m_{i}=\frac{d_{i} z_{i} F}{R T}
$$

where $z_{i} F$ is the charge carried by a mole of species $A_{i}, R$ is the universal gas constant and $T$ is the local temperature.

We consider the system to involve one-dimensional steady-state transport of the various species to a rotating disk electrode with simultaneous homo-

geneous reaction. Furthermore, the homogeneous reactions are assumed to have much more rapid kinetics than the electrode reactions and to instantaneously reach thermodynamic equilibrium. Edge and double-layer effects are not included. Accordingly, the transport equation for each species becomes

$$
-d_{i} \Delta w_{i}+\operatorname{div}\left(v w_{i}\right)-m_{i} \operatorname{div}\left(w_{i} \nabla \Phi\right)=S_{i}
$$


The species must also satisfy the electroneutrality condition everywhere in the system, i.e.

$$
\sum_{i=1}^{N S} z_{i} w_{i}=0
$$

where $N S$ is the number of soluble species.

The solution of the governing differential equations together with the algebraic equations for homogeneous equilibrium requires first combining the transport equation for the individual species so as to eliminate the homogeneous reaction rate terms. The resulting equations which depend on the stoichiometry of the homogeneous reactions amount to mass balances on different components of the system (nickel, iron, hydrogen, etc. ). In this analysis, we consider a problem of the codeposition of nickel and iron from a sulfate solution. This particular codeposition process is important because it permits the fabrication of magnetic devices from permalloy. For this nickeliron sulfate bath $\mathrm{NiSO}_{4}, \mathrm{FeSO}_{4}$ and $\mathrm{H}_{3} \mathrm{BO}_{3}$ are dissolved in water and $\mathrm{H}_{2} \mathrm{SO}_{4}$ is used for $\mathrm{pH}$ adjustment. Consequently, the five dissolved species considered are $\mathrm{Ni}^{2+}, \mathrm{Fe}^{2+}, \mathrm{H}^{+}, \mathrm{SO}_{4}^{2-}$ and $\mathrm{HSO}_{4}^{-}$. The metals do not form complexes with sulfate. The only homogeneous reaction considered is that between $\mathrm{SO}_{4}^{2-}$ and $\mathrm{HSO}_{4}^{-}$

$$
\mathrm{SO}_{4}^{2-}+\mathrm{H}^{+} \rightleftharpoons{ }_{k_{-1}}^{k_{1}} \mathrm{HSO}_{4}^{-}
$$

This reaction is commonly described as bimolecular in the forward direction and monomolecular in the backward direction. With $k_{1}$ and $k_{-1}$ the reaction rate constants of the forward and backward reaction.

In this case, the homogeneous reaction term in 4 takes the form

$$
\begin{aligned}
S_{3} & =-k_{1} w_{3} w_{4}+k_{-1} w_{5} \\
S_{4} & =-k_{1} w_{3} w_{4}+k_{-1} w_{5} \\
S_{5} & =k_{1} w_{3} w_{4}-k_{-1} w_{5}
\end{aligned}
$$

for all ions involved in reaction 6 .

$K=\frac{k_{-1}}{k_{1}}$ denotes the equilibrium constant for homogeneous reaction and $w_{i}(1 \leq i \leq 5)$ are respectively the concentrations of $\mathrm{Ni}^{2+}, \mathrm{Fe}^{2+}, \mathrm{H}^{+}, \mathrm{HSO}_{4}^{-}$ and $S O_{4}^{2-}$. 
As mentioned previously, cathode reactions at the electrodeposition surface will involve the doubly-charged metal ions. During NiFe codeposition, we consider the following two electrode reactions

$$
\begin{aligned}
& N i^{2+}+2 e^{-} \rightarrow N i \\
& F e^{2+}+2 e^{-} \rightarrow F e
\end{aligned}
$$

All the species are considered to be inert with regard to cathode reactions, with the exception of hydrogen. The formation of hydrogen is involved only in one cathode reaction from the protium cation,

$$
2 \mathrm{H}^{+}+2 e^{-} \rightarrow \mathrm{H}_{2}
$$

The rates associated with these fundamental reactions can all be expressed in terms of local potential $V$, and local ion species concentrations $w_{i}$. Fluxes at the cathode surface $(y=0)$ are given by [9], [7]

$$
J_{k}(0)=\frac{i_{k}}{2 F}=-\beta_{k} w_{k}(0) \exp \left[-\alpha_{k} z_{k} \frac{F V}{R T}\right]
$$

where $i_{k} \quad k=1,2,3$ are the current densities for reactions $(7),(8),(9), V$ is the cathode potential on the surface hydrolysis electrode (SHE) scale after ohmic correction and $\beta_{k}, \alpha_{k}$ are respectively the rate constants and transfer coefficients. The rate relations are commonly known as Butler-Volmer equations. Values of the constants in these relations are widely publish [9], [7].

The three partial currents given in equations (10), (11), (12) are of practical importance because they are directly related to the deposition rates of nickel and iron and to the rate of hydrogen generation. The latter is important when ion fluxes are comparable to the diffusion limited ion transport rates since hydrogen that can not be diffused from the cathode surface will lead to hydrogen bubble formation. Hydrogen bubbles that do not detach from the cathode give rise to poor morphology of the deposited metal. Moreover, even high concentrations of hydrogen may lead to poor metal properties as hydrogen may become entrapped in the metal during the deposition process.

The bulk region considered is a domain $\Omega$ and $\delta$ is the hydrodynamic boundary layer thickness. As mentioned elsewhere [17], we consider the bulk region to be fully established beyond a distance $3 \delta$ from the electrode surface. 
The presence of boric acid has not been explicitly included in this model. Over the normal range of operating conditions for NiFe codeposition, this species remains undissociated and consequently is transported by diffusion and convection, but not by migration. In addition, it is not known to participate in any homogeneous or electrochemical reaction. Thus, the transport of $\mathrm{H}_{3} \mathrm{BO}_{3}$ is completely decoupled from that of the other dissolved species. As proposed by Horkans [8], the role it plays in codeposition may be to adsorb onto the electrode surface and modify the kinetics of nickel and iron deposition. It is beyond the scope of this analysis to account for this effect in a detailed explicit way.

Boundary conditions have to be added as follows :

$$
\begin{aligned}
& \left\{\begin{array}{l}
-d_{i} w_{i}^{\prime}(0)-m_{i} w_{i}(0) \Phi^{\prime}(0)=-\beta_{i} w_{i}(0) \exp \left[-\alpha_{i} z_{i} F V / R T\right] \text { for } 1 \leq i \leq 3 \\
w_{4}(0)=w_{5}(0)=0 \\
\Phi(0)=V
\end{array}\right. \\
& \left\{\begin{array}{l}
w_{i}(3 \delta)=w_{i}^{*} \\
\Phi(3 \delta)=0
\end{array} \quad \text { for } 1 \leq i \leq 5\right.
\end{aligned}
$$

where $V \leq 0, w_{i}^{*}$ represents the total amount of component $A_{i}$ added to the bulk solution.

Let us also include electroneutrality condition (5) and the homogeneous equilibria (6), as well as the following material balance expressions

$$
\begin{aligned}
w_{1}^{*} & =w_{1}^{T} \\
w_{2}^{*} & =w_{2}^{T} \\
w_{4}^{*}+w_{5}^{*} & =w_{5}^{T} \\
2 w_{1}^{*}+2 w_{2}^{*}+w_{3}^{*}-2 w_{4}^{*}-w_{5}^{*} & =0 \\
w_{5}^{*} & =K w_{3}^{*} w_{4}^{*}
\end{aligned}
$$

where $w_{i}^{T}$ represents the total amount of component $i$ added to the bulk solution and $K=\frac{k_{-1}}{k_{1}}$. One can deduce easily

$$
\begin{aligned}
& w_{4}^{*}=w_{5}^{T}-w_{5}^{*} \\
& w_{3}^{*}=-2\left(w_{1}^{T}+w_{2}^{T}\right)+2 w_{5}^{T}-w_{5}^{*} \\
& k w_{5}^{* 2}-\left(1+K\left(w_{1}^{T}+w_{2}^{T}\right)+w_{5}^{T}\right) w_{5}^{*}+2 K w_{5}^{T}\left(w_{5}^{T}-w_{1}^{T}-w_{2}^{T}\right)=0
\end{aligned}
$$


Now we are able to give the system satisfied by the concentrations of different species that are involved in our model

$$
\begin{cases}-d_{i} w_{i}^{\prime \prime}+b_{i}(y) w_{i}^{\prime}-m_{i}\left(w_{i} \Phi^{\prime}\right)^{\prime}=0 \quad \text { in } \Omega & \text { for } i=1,2 \\ -d_{i} w_{i}^{\prime \prime}+b_{i}(y) w_{i}^{\prime}-m_{i}\left(w_{i} \Phi^{\prime}\right)^{\prime}=S_{i} \quad \text { in } \Omega \quad \text { for } i=3,4,5 & \\ 2 w_{1}+2 w_{2}+w_{3}-w_{4}-2 w_{5}=0 \quad \text { in } \Omega & \\ w_{i}(3 \delta)=w_{i}^{*} \quad \text { for } 1 \leq i \leq 5 & \\ -d_{i} w_{i}^{\prime}(0)-m_{i} w_{i}(0) \Phi^{\prime}(0)=-\beta_{i} w_{i}(0) \exp \left[-\alpha_{i} z_{i} \frac{F V}{R T}\right] & \text { for } 1 \leq i \leq 5 \\ \Phi(0)=V ; \Phi(3 \delta)=0 ; & \end{cases}
$$

where $b_{i}(y)=v_{y}$ and $\beta_{4}=\beta_{5}=0$.

Finally let's examine the expression of the velocity of the electrolyte $v$. Cochran and Von Karman [10], [1] obtained that the fluid velocity component $v_{y}$ of $v$ can be expressed in term of power series expansion that involves a reduced variable $\gamma=y \sqrt{\frac{\omega}{\nu}}$ :

$$
v_{y}=\sqrt{\nu \omega} H(\gamma)=\sqrt{\nu \omega}\left(-a \gamma^{2}+\frac{1}{3} \gamma^{3}+\frac{b}{6} \gamma^{4}+\ldots\right)
$$

where $a=0.51023, \omega$ is the rotation speed of the $R D E$, and $\nu$ is the kinematic viscosity of the solution. The expansion also yields the boundary layer thickness $\delta$,

$$
\delta=\left(\frac{3 d}{a}\right)^{\frac{1}{3}} \omega^{-\frac{1}{2}} v^{\frac{1}{6}}
$$

where $d$ is a reference diffusion coefficient taken to correspond to the species in solution with the smallest diffusion coefficient.

\section{$3 \quad$ Numerical analysis of the model}

For $x \in[0,3 \delta]$, we put

$$
\psi(x)=\Phi(x)-\frac{V}{3 \delta}(3 \delta-x)
$$


and for $i=1, \ldots, 5$,

$$
\begin{aligned}
& v_{i}(x)=w_{i}(x)-w_{i}^{*} \\
& m_{i}=\frac{F}{R T} d_{i} z_{i} \\
& \gamma_{i}=\beta_{i} \exp \left(-\alpha_{i} \frac{m_{i}}{d_{i}}\right) \\
& b_{i}=b .
\end{aligned}
$$

System (13) is then equivalent to the two following systems :

$$
\left\{\begin{array}{l}
-d_{i} v_{i}^{\prime \prime}+\left(b(y)+m_{i} \frac{V}{3 \delta}\right) v_{i}^{\prime}-m_{i}\left(v_{i} \psi^{\prime}\right)^{\prime}=m_{i} w_{i}^{*} \psi^{\prime \prime}+\widetilde{S}_{i} \quad \text { in }(0,3 \delta) \\
-d_{i} v_{i}^{\prime}(0)-m_{i}\left(v_{i}(0)+w_{i}^{*}\right)\left(\psi^{\prime}(0)-\frac{V}{3 \delta}\right)=-\gamma_{i}\left(v_{i}(0)+w_{i}^{*}\right) \\
v_{i}(3 \delta)=0, \quad \text { for } 1 \leq i \leq 5
\end{array}\right.
$$

and

$$
\left\{\begin{array}{l}
-\left[\sum_{i=1}^{5} z_{i} m_{i}\left(v_{i}+w_{i}^{*}\right) \psi^{\prime}\right]^{\prime}=\sum_{i=1}^{5} z_{i} d_{i} v_{i}^{\prime \prime}-\frac{V}{3 \delta} \sum_{i=1}^{5} z_{i} m_{i} v_{i}^{\prime} \quad \text { in }(0,3 \delta) \\
\psi(0)=\psi(3 \delta)=0
\end{array}\right.
$$

with

$$
\begin{aligned}
& \widetilde{S}_{1}=\widetilde{S}_{2}=0 \\
& \widetilde{S}_{3}:=\widetilde{S}_{3}\left(v_{3}, v_{4}, v_{5}\right)=-k_{1}\left(v_{3}+w_{3}^{*}\right)\left(v_{4}+w_{4}^{*}\right)+k_{-1}\left(v_{3}+w_{3}^{*}\right) \\
& \widetilde{S}_{4}=\widetilde{S}_{3} \\
& \widetilde{S}_{5}=-\widetilde{S}_{3} .
\end{aligned}
$$

In order to solve the coupled system (16) and (17) we consider a fixed point method with respect to the reaction term (18). That means : at each iteration we consider $\widetilde{S}_{i}$ given and at each steep of the fixed point method we solve a coupled systems of the type :

$$
\left\{\begin{array}{l}
-d_{i} v_{i}^{\prime \prime}+\left(b(y)+m_{i} \frac{V}{3 \delta}\right) v_{i}^{\prime}-m_{i}\left(v_{i} \Psi^{\prime}\right)^{\prime}=m_{i} w_{i}^{*} \Psi^{\prime \prime}+f_{i} \text { in }(0,3 \delta), \\
-d_{i} v_{i}^{\prime}(0)-m_{i}\left(v_{i}(0)+w_{i}^{*}\right)\left(\psi^{\prime}(0)-\frac{V}{3 \delta}\right)=-\gamma_{i}\left(v_{i}(0)+w_{i}^{*}\right), \\
v_{i}(3 \delta)=0, \text { for } 1 \leq i \leq 5
\end{array}\right.
$$

and

$$
\left\{\begin{array}{l}
-\left[\sum_{i=1}^{5} \varepsilon_{i} \psi^{\prime}\right]^{\prime}=\sum_{i=1}^{5} g_{i} \quad \text { in }(0,3 \delta) \\
\psi(0)=0, \psi(3 \delta)=0
\end{array}\right.
$$

with $\left(f_{i}, \varepsilon_{i}\right) \in\left(C^{1}([0,3 \delta])\right)^{2}, g_{i} \in C([0,3 \delta])$ and $\Psi \in C^{2}([0,3 \delta])$. The functions $f_{i}, \varepsilon_{i}$ and $g_{i}$ depends on the numerical method to solve (16) and (17) 
and it change at each iteration.

To show the existence and uniqueness of the solution of system (19) we consider a variational formulation.

Let

$$
H=\left\{\varphi \in H^{1}(0,3 \delta) / \varphi(3 \delta)=0\right\}
$$

and we define the norm $\|\cdot\|_{H}$ in $H$ by :

$$
\|\varphi\|_{H}=\left(\int_{0}^{3 \delta}\left(\varphi^{\prime}\right)^{2}(x) d x\right)^{\frac{1}{2}} .
$$

Let $a_{i}\left(v_{i}, \varphi\right)$ be the following bilinear form :

$$
\begin{aligned}
a_{i}\left(v_{i}, \varphi\right)= & \left(\gamma_{i}+m_{i} \frac{V}{3 \delta}\right) v_{i}(0) \varphi(0)+d_{i} \int_{0}^{3 \delta} v_{i}^{\prime}(x) \varphi^{\prime}(x) d x \\
& +\int_{0}^{3 \delta}\left(b(x)+m_{i} \frac{V}{3 \delta}\right) v_{i}^{\prime}(x) \varphi(x) d x+m_{i} \int_{0}^{3 \delta} v_{i}(x) \Psi^{\prime}(x) \varphi^{\prime}(x) d x .
\end{aligned}
$$

By multiplying the first equation of (19) by $\varphi \in H$ and integrate by part we obtain the weak formulation

$$
a_{i}\left(v_{i}, \varphi\right)=-\left(\gamma_{i}+m_{i} \frac{V}{3 \delta}\right) w_{i}^{*} \varphi(0)-m_{i} \int_{0}^{3 \delta} w_{i}^{*} \Psi^{\prime}(x) \varphi^{\prime}(x) d x+\int_{0}^{3 \delta} f_{i}(x) \varphi(x) d x
$$

Definition 1 We say that $\left(v_{1}, v_{2}, v_{3}, v_{4}, v_{5}\right)$ is a solution of (19) if and only if

$$
\left\{\begin{aligned}
\left(v_{1}, v_{2}, v_{3}, v_{4}, v_{5}\right) \in(H)^{5}, & \\
a_{i}\left(v_{i}, \varphi\right)= & -\left(\gamma_{i}+m_{i} \frac{V}{3 \delta}\right) w_{i}^{*} \varphi(0)-m_{i} \int_{0}^{3 \delta} w_{i}^{*} \Psi^{\prime}(x) \varphi^{\prime}(x) d x \\
& +\int_{0}^{3 \delta} f_{i}(x) \varphi(x) d x \quad \text { for } \varphi \in H \quad \text { and } \quad 1 \leq i \leq 5 .
\end{aligned}\right.
$$

Let $u=\max _{1 \leq i \leq 5}\left|m_{i}\right|, d=\min _{1 \leq i \leq 5}\left|d_{i}\right|, \eta=\max \left(\left\|\Psi^{\prime}\right\|_{\infty},\left\|\Psi^{\prime \prime}\right\|_{\infty}\right)$ and $\varepsilon=d-a(3 \delta)^{2}+\frac{u V}{2}-\frac{3 \delta u \eta}{2}(3 \delta+1)$.

Proposition 2 Suppose that $\varepsilon>0$. Then problem (19) has a unique solution in $C^{2}([0,3 \delta])$. 
Proof. First we will prove that the form $a_{i}$ is bilinear continuous on $H \times H$, for $1 \leq i \leq 5$. It is clair that the form $a_{i}(.,$.$) is bilinear. In the other hand$ for all $v \in H$ we have

$$
v(x)=\int_{3 \delta}^{x} v^{\prime}(t) d t
$$

By the Hölder's Inequality we deduce

$$
\|v\|_{L^{2}(0,3 \delta)} \leq 3 \delta\left\|v^{\prime}\right\|_{L^{2}(0,3 \delta)} \forall v \in H
$$

and

$$
\|v\|_{L^{\infty}(0,3 \delta)} \leq \sqrt{3 \delta}\left\|v^{\prime}\right\|_{L^{2}(0,3 \delta)} \forall v \in H
$$

then

$$
\|v\|_{L^{1}(0,3 \delta)} \leq(3 \delta)^{3 / 2}\left\|v^{\prime}\right\|_{L^{2}(0,3 \delta)} \forall v \in H .
$$

Using (23), (27) and the Hölder's Inequality, we obtain

$$
\begin{aligned}
\left|a_{i}(v, \varphi)\right| \leq & 3 \delta\left|\gamma_{i}+m_{i} \frac{V}{3 \delta}\right|\left\|v^{\prime}\right\|_{L^{2}(0,3 \delta)}\left\|\varphi^{\prime}\right\|_{L^{2}(0,3 \delta)} \\
& +d_{i}\left\|v^{\prime}\right\|_{L^{2}(0,3 \delta)}\left\|\varphi^{\prime}\right\|_{L^{2}(0,3 \delta)} \\
& +\left\|b+m_{i} \frac{V}{3 \delta}\right\|_{L^{\infty}(0,3 \delta)}\left\|v^{\prime}\right\|_{L^{2}(0,3 \delta)}\|\varphi\|_{L^{2}(0,3 \delta)} \\
& +\mid m_{i}\left\|\Psi^{\prime}\right\|_{L^{\infty}(0,3 \delta)}\|v\|_{L^{2}(0,3 \delta)}\left\|\varphi^{\prime}\right\|_{L^{2}(0,3 \delta)}
\end{aligned}
$$

For $x \in[0,3 \delta]$, we have $b(x)=-a x^{2}$ and $V \leq 0$, then

$$
\left\|b+m_{i} \frac{V}{3 \delta}\right\|_{L^{\infty}(0,3 \delta)} \leq a(3 \delta)^{2}-u \frac{V}{3 \delta},
$$

It follows from (26), (29) and (30) that

$$
\left|a_{i}(v, \varphi)\right| \leq\left(a(3 \delta)^{3}+3 \delta \gamma_{i}+3 \delta u \eta-2 u V+d_{i}\right)\left\|v^{\prime}\right\|_{L^{2}(0,3 \delta)}\left\|\varphi^{\prime}\right\|_{L^{2}(0,3 \delta)},
$$

then

$$
\left|a_{i}(v, \varphi)\right| \leq\left(a(3 \delta)^{3}+3 \delta \gamma_{i}+3 \delta u \eta-2 u V+d_{i}\right)\left\|v^{\prime}\right\|_{H}\left\|\varphi^{\prime}\right\|_{H},
$$

which proves that the form $a_{i}$ is continuous in $H \times H$.

Second we will prove that the form $a_{i}$ is coercive on $H \times H$. 
For all $(v, \varphi) \in H \times H$, we have

$$
\begin{aligned}
a_{i}(v, \varphi)= & \left(\gamma_{i}+m_{i} \frac{V}{3 \delta}\right) v(0) \varphi(0)+d_{i} \int_{0}^{3 \delta} v^{\prime}(x) \varphi^{\prime}(x) d x \\
& +\frac{1}{2} \int_{0}^{3 \delta}\left(b(x)+m_{i} \frac{V}{3 \delta}\right) v^{\prime}(x) \varphi(x) d x \\
& +\frac{1}{2} \int_{0}^{3 \delta}\left(b(x)+m_{i} \frac{V}{3 \delta}\right) v^{\prime}(x) \varphi(x) d x \\
& +\frac{1}{2} m_{i} \int_{0}^{3 \delta} v(x) \Psi^{\prime}(x) \varphi^{\prime}(x) d x \\
& +\frac{1}{2} m_{i} \int_{0}^{3 \delta} v(x) \Psi^{\prime}(x) \varphi^{\prime}(x) d x .
\end{aligned}
$$

By integration by part we obtain

$$
\begin{aligned}
a_{i}(v, \varphi)= & d_{i} \int_{0}^{3 \delta} v^{\prime}(x) \varphi^{\prime}(x) d x+\frac{1}{2} \int_{0}^{3 \delta}\left(b(x)+m_{i} \frac{V}{3 \delta}\right) v^{\prime}(x) \varphi(x) d x \\
& +\frac{1}{2} m_{i} \int_{0}^{3 \delta} v(x) \Psi^{\prime}(x) \varphi^{\prime}(x) d x \\
& +\left(\gamma_{i}+m_{i} \frac{V}{3 \delta}\right) v(0) \varphi(0)-\frac{1}{2} m_{i} \frac{V}{3 \delta} v(0) \varphi(0) \\
& -\frac{1}{2} \int_{0}^{3 \delta}\left(\left(b(x)+m_{i} \frac{V}{3 \delta}\right) \varphi(x)\right)^{\prime} v(x) d x \\
& -\frac{1}{2} m_{i} v(0) \varphi(0) \Psi^{\prime}(0)-\frac{1}{2} m_{i} \int_{0}^{3 \delta} \varphi(x)\left(\Psi^{\prime} v\right)^{\prime}(x) d x .
\end{aligned}
$$

It follows that

$$
\begin{aligned}
a_{i}(v, \varphi)= & \left\{\gamma_{i}+\frac{m_{i}}{2}\left(\frac{V}{3 \delta}-\Psi^{\prime}(0)\right)\right\} v(0) \varphi(0) \\
& +d_{i} \int_{0}^{3 \delta} v^{\prime}(x) \varphi^{\prime}(x) d x-\frac{1}{2} \int_{0}^{3 \delta}\left(b^{\prime}+m_{i} \Psi^{\prime \prime}\right)(x) v(x) \varphi(x) d x \\
& +\frac{1}{2} \int_{0}^{3 \delta}\left[\left(b+m_{i} \frac{V}{3 \delta}-m_{i} \Psi^{\prime}\right)\left(v^{\prime} \varphi-v \varphi^{\prime}\right)\right](x) d x .
\end{aligned}
$$

Then

$$
\begin{aligned}
a_{i}(v, v)= & \left\{\gamma_{i}+\frac{m_{i}}{2}\left(\frac{V}{3 \delta}-\Psi^{\prime}(0)\right)\right\} v^{2}(0)+d_{i} \int_{0}^{3 \delta} v^{\prime 2}(x) d x \\
& -\frac{1}{2} \int_{0}^{3 \delta}\left(b^{\prime}+m_{i} \Psi^{\prime \prime}\right)(x) v^{2}(x) d x .
\end{aligned}
$$


It follows that

$$
\begin{aligned}
a_{i}(v, v) \geq & \gamma_{i} v^{2}(0)-\left|\frac{m_{i}}{2}\left(\frac{V}{3 \delta}-\Psi^{\prime}(0)\right)\right| v^{2}(0)+d_{i} \int_{0}^{3 \delta} v^{\prime 2}(x) d x \\
& -\left|\frac{1}{2} \int_{0}^{3 \delta}\left(b^{\prime}+m_{i} \Psi^{\prime \prime}\right)(x) v^{2}(x) d x\right| .
\end{aligned}
$$

Therefore by the fact that $\gamma_{i} \geq 0$ and by (26), (27), we deduce

$$
\begin{aligned}
a_{i}(v, v) \geq & -\left|\frac{m_{i}}{2}\left(\frac{V}{3 \delta}-\Psi^{\prime}(0)\right)\right| 3 \delta\left\|v^{\prime}\right\|_{L^{2}(0,3 \delta)}^{2}+d_{i}\left\|v^{\prime}\right\|_{L^{2}(0,3 \delta)}^{2} \\
& -\frac{1}{2}(3 \delta)^{2}\left\|b^{\prime}+m_{i} \Psi^{\prime \prime}\right\|_{L^{\infty}(0,3 \delta)}\left\|v^{\prime}\right\|_{L^{2}(0,3 \delta)}^{2} .
\end{aligned}
$$

Or $\left\|\Psi^{\prime}\right\|_{\infty}<\eta,\left\|\Psi^{\prime \prime}\right\|_{\infty}<\eta$ and $\left\|b^{\prime}\right\|_{\infty}=6 a \delta$, it follows that

$$
a_{i}(v, v) \geq\left[d_{i}-a(3 \delta)^{2}+\frac{u V}{2}-\frac{3 \delta u \eta}{2}(3 \delta+1)\right]\left\|v^{\prime}\right\|_{L^{2}(0,3 \delta)}^{2} .
$$

Then

$$
a_{i}(v, v) \geq \varepsilon\left\|v^{\prime}\right\|_{L^{2}(0,3 \delta)}^{2} .
$$

Which means that the form $a_{i}$ is coercive on $H \times H$.

Now let prove that the form $F_{i}$ defined by

$$
F_{i}(\varphi)=-\left(\gamma_{i}+m_{i} \frac{V}{3 \delta}\right) w_{i}^{*} \varphi(0)-m_{i} \int_{0}^{3 \delta} w_{i}^{*} \Psi^{\prime}(x) \varphi^{\prime}(x) d x+\int_{0}^{3 \delta} f_{i}(x) \varphi(x) d x
$$

for $\varphi \in H$ is continuous.

We have

$$
\begin{aligned}
\left|F_{i}(\varphi)\right| \leq & \left(\gamma_{i}+u \frac{V}{3 \delta}\right) w_{i}^{*}\|\varphi\|_{L^{\infty}(0,3 \delta)}+u w_{i}^{*}\left\|\Psi^{\prime}\right\|_{L^{\infty}(0,3 \delta)}\|\varphi\|_{L^{1}(0,3 \delta)} \\
& \left.+\left\|f_{i}\right\|_{L^{\infty}(0,3 \delta)}\|\varphi\|_{L^{1}(0,3 \delta}\right)
\end{aligned}
$$

It follows from (27) and (28) that

$$
\left|F_{i}(\varphi)\right| \leq 3 \delta\left\{\left(\gamma_{i}+u \frac{V}{3 \delta}\right) w_{i}^{*}+u w_{i}^{*} \eta(3 \delta)+(3 \delta)^{2}\left\|f_{i}\right\|_{H}\right\}\|\varphi\|_{H},
$$

therefore $F_{i}$ is continuous on $H$.

Then by the Lax-Milgram Theorem we deduce that problem (25) has a solution $\left(v_{1}, v_{2}, v_{3}, v_{4}, v_{5}\right) \in(H)^{5}$. Finally let prove that $\left(v_{1}, v_{2}, v_{3}, v_{4}, v_{5}\right) \in$ $\left(C^{2}([0,3 \delta])\right)^{5}$.

We note by $1_{(0,3 \delta]}$ the function defined on $\mathbb{R}$ by

$$
1_{(0,3 \delta]}(x)=\left\{\begin{array}{ll}
1 & \text { if } \\
0 & \text { ifnot. }
\end{array} \quad x \in(0,3 \delta]\right.
$$


By (24), for all $\varphi \in H^{1}(] 0,3 \delta[)$, we have

$$
\begin{aligned}
& \int_{0}^{3 \delta}\left[d_{i} v_{i}^{\prime}+m_{i}\left(v_{i}+w_{i}^{*}\right) \Psi^{\prime}\right](x) \varphi^{\prime}(x) d x \\
& =\int_{0}^{3 \delta}\left[f_{i}-\left(b+m_{i} \frac{V}{3 \delta}\right) v_{i}^{\prime}-\frac{1}{3 \delta}\left(\gamma_{i}+m_{i} \frac{V}{3 \delta}\right)\left(w_{i}^{*}+v_{i}\right)\left(1-1_{(0,3 \delta]}\right)\right](x) \varphi(x) d x .
\end{aligned}
$$

Then by the fact that

$$
\left[f_{i}-\left(b+m_{i} \frac{V}{3 \delta}\right) v_{i}^{\prime}-\frac{1}{3 \delta}\left(\gamma_{i}+m_{i} \frac{V}{3 \delta}\right)\left(w_{i}^{*}+v_{i}\right)\left(1-1_{(0,3 \delta]}\right)\right] \in L^{2}((0,3 \delta)),
$$

it follows that

$$
\left[d_{i} v_{i}^{\prime}+m_{i}\left(v_{i}+w_{i}^{*}\right) \Psi^{\prime}\right] \in H^{1}((0,3 \delta)) .
$$

Therefore $v_{i}^{\prime} \in H^{1}((0,3 \delta))$, then $v_{i}^{\prime} \in C([0,3 \delta])$, which implies that

$$
v_{i} \in C^{1}([0,3 \delta]) .
$$

Or $\Psi^{\prime} \in C^{1}([0,3 \delta])$, then $v_{i}^{\prime} \in C^{1}([0,3 \delta])$, which implies that

$$
v_{i} \in C^{2}([0,3 \delta]) \text {. }
$$

and proposition 2 follows.

With a similar method as proposition 2 we show the following result :

Proposition 3 Suppose that there exists $k>0$ such that for all $x \in[0,3 \delta]$, $\sum_{i=1}^{5} \varepsilon_{i}(x) \geq k$. Then problem (20) has a unique solution in $C^{2}([0,3 \delta])$.

\subsection{Domain decomposition}

\subsubsection{Concentrations}

The domain decomposition algorithm considered here has previously been used and analyzed by F. Gastaldi, L. Gastaldi and A. Quarteroni (see [5]), in the case of constant coefficients. We have generalized the Gastaldi- Quarteroni's iterative substructuring method to the case with non constant coefficients and Robin boundary conditions in $x=0$.

System (16) is equivalent to the following system :

$$
\left\{\begin{array}{l}
-d_{i} v_{i}^{\prime \prime}+\left(b(y)+m_{i} \frac{V}{3 \delta}\right) v_{i}^{\prime}=m_{i}\left[\left(v_{i}+w_{i}^{*}\right) \psi^{\prime}\right]^{\prime}+\widetilde{S}_{i} \quad \text { in }(0,3 \delta), \\
v_{i}(3 \delta)=0, \\
-d_{i} v_{i}^{\prime}(0)=\left[m_{i}\left(\psi^{\prime}(0)-\frac{V}{3 \delta}\right)-\gamma_{i}\right]\left(v_{i}(0)+w_{i}^{*}\right) \quad \text { for } \quad 1 \leq i \leq 5
\end{array}\right.
$$


For $1 \leq i \leq 5$ we put :

$$
\left\{\begin{array}{l}
B_{0}^{i}=b+m_{i} \frac{V}{3 \delta} . \\
F_{i}\left(v_{1}, v_{2}, v_{3}, v_{4}, v_{5}, \psi\right)=m_{i}\left[\left(v_{i}+w_{i}^{*}\right) \psi^{\prime}\right]^{\prime}+\widetilde{S}_{i} . \\
G_{i}\left(v_{i}, \psi\right)(0)=\left[m_{i}\left(\psi^{\prime}(0)-\frac{V}{3 \delta}\right)-\gamma_{i}\right]\left(v_{i}(0)+w_{i}^{*}\right) .
\end{array}\right.
$$

Then the system (45) comes

$$
\left\{\begin{array}{l}
-d_{i} v_{i}^{\prime \prime}+B_{0}^{i}(y) v_{i}^{\prime}=F_{i}\left(v_{1}, v_{2}, v_{3}, v_{4}, v_{5}, \psi\right) \quad \text { in }(0,3 \delta), \\
v_{i}(3 \delta)=0, \\
-d_{i} v_{i}^{\prime}(0)=G_{i}\left(v_{i}, \psi\right)(0) \quad \text { for } 1 \leq i \leq 5
\end{array}\right.
$$

We note

$$
L^{i} v=-d_{i} v_{i}^{\prime \prime}+B_{0}^{i} v_{i}^{\prime}, \quad \text { for } \quad 1 \leq i \leq 5 .
$$

For the resolution of the system (47), we will proceed in two steps :

\section{Initialization}

Let $v_{i, 0}$ be the solution of the homogeneous system :

$$
\left\{\begin{array}{l}
L^{i} v_{i}=0 \quad \text { in }(0,3 \delta), \\
v_{i}(3 \delta)=0, \\
G_{i}\left(v_{i}, \psi\right)(0)=-d_{i} v_{i}^{\prime}(0), \quad \text { for } 1 \leq i \leq 5 .
\end{array}\right.
$$

External loop: for $n \in \mathbb{N}, v_{i, n+1}$ we solve the linear system :

$$
\left\{\begin{array}{l}
L^{i} v_{i}=F_{i}\left(v_{1, n}, v_{2, n}, v_{3, n}, v_{4, n}, v_{5, n}, \psi\right) \quad \text { in }(0,3 \delta), \\
v_{i}(3 \delta)=0, \quad \text { for } \quad 1 \leq i \leq 5 . \\
G_{i}\left(v_{i}, \psi\right)(0)=-d_{i} v_{i, n}^{\prime}(0), \quad
\end{array}\right.
$$

and the potential equation(17). The system (17) is equivalent to the following system :

$$
\left\{\begin{array}{l}
-\left[p \psi^{\prime}\right]^{\prime}=q \quad \text { in }(0,3 \delta) \\
\psi(0)=\psi(3 \delta)=0
\end{array}\right.
$$

with

$$
\left\{\begin{array}{l}
p=\sum_{i=1}^{5} z_{i} m_{i}\left(v_{i, n}+w_{i}^{*}\right) \\
q=\sum_{i=1}^{5} z_{i} d_{i} v_{i, n}^{\prime \prime}-\frac{V}{3 \delta} \sum_{i=1}^{5} z_{i} m_{i} v_{i, n}^{\prime}
\end{array}\right.
$$

Internal loop: Resolution of system (50) by an iterative methods on subdomain.

Let

$$
n \in \mathbb{N} \text { and } c \in(0,3 \delta) \text { fixed. }
$$


Let $A$ and $B$ two real parameters such that $A B \leq 0, A \neq B, v_{i 1}^{0}=v_{i 2}^{0}=v_{i, n}$ and

$$
\lambda_{i}^{0}=d_{i}\left(v_{i 2}^{0}\right)^{\prime}(c)-\left(\frac{1}{2} B(c)+A\right) v_{i 2}^{0}(c),
$$

solve for $k \geq 0$

$$
\left\{\begin{array}{l}
L^{i} v_{i 1}^{k+1}=F_{i}\left(v_{1, n}, v_{2, n}, v_{3, n}, v_{4, n}, v_{5, n}, \psi\right) \quad \text { in }(0, c) \\
d_{i}\left(v_{i 1}^{k+1}\right)^{\prime}(c)-\left(\frac{1}{2} B_{0}(c)+A\right) v_{i 1}^{k+1}(c)=\lambda_{i}^{k} \\
G_{i}\left(v_{i 1}^{k+1}, \psi\right)(0)=-d_{i}\left(v_{i, n}\right)^{\prime}(0)
\end{array}\right.
$$

then

$$
\left\{\begin{array}{l}
L^{i} v_{i 2}^{k+1}=F_{i}\left(v_{1, n}, v_{2, n}, v_{3, n}, v_{4, n}, v_{5, n}, \psi\right) \quad \text { in }(c, 3 \delta) \\
d_{i}\left(v_{i 2}^{k+1}\right)^{\prime}(c)-\left(\frac{1}{2} B_{0}(c)+B\right) v_{i 2}(c)= \\
d_{i}\left(v_{i 1}^{k+1}\right)^{\prime}(c)-\left(\frac{1}{2} B_{0}(c)+B\right) v_{i 1}^{k+1}(c), \\
v_{i 2}^{k+1}(3 \delta)=0,
\end{array}\right.
$$

and finally set

$$
\lambda_{i}^{k+1}=d_{i}\left(v_{i 2}^{k+1}\right)^{\prime}(c)-\left(\frac{1}{2} B(c)+A\right) v_{i 2}^{k+1}(c) .
$$

\subsubsection{Resolution of the potential equation}

For the resolution of the system (51), we use the same iterative subdomain method.

Let $c$ the real given by (53), $A^{\prime}, B^{\prime}$ two real parameters such that $A^{\prime} B^{\prime} \leq 0$ and $A^{\prime} \neq B^{\prime}$, Given $\beta^{0}$.

Solve for $k \geq 0$

$$
\left\{\begin{array}{l}
-\left[p\left(w+v^{*}\right)\left(\psi_{1}^{k+1}\right)^{\prime}\right]^{\prime}=q\left(w+v^{*}\right) \quad \text { in } H^{1}(0, c) \\
\psi_{1}^{k+1}(0)=0 \\
{\left[p\left(w+v^{*}\right)\left(\psi_{1}^{k+1}\right)^{\prime}\right](c)-A^{\prime} \psi_{1}^{k+1}(c)=\beta^{k}}
\end{array}\right.
$$

then

$$
\left\{\begin{array}{l}
-\left[p\left(w+v^{*}\right)\left(\psi_{2}^{k+1}\right)^{\prime}\right]^{\prime}=q\left(w+v^{*}\right) \quad \text { in } H^{1}(c, \delta), \\
\psi_{2}^{k+1}(\delta)=0, \\
{\left[p\left(w+v^{*}\right)\left(\psi_{2}^{k+1}\right)^{\prime}\right](c)-B^{\prime} \psi_{2}^{k+1}(c)=\left[p\left(w+v^{*}\right)\left(\psi_{1}^{k+1}\right)^{\prime}\right](c)-B^{\prime} \psi_{1}^{k+1}(c),}
\end{array}\right.
$$

and finally set

$$
\beta^{k+1}=\left[p\left(w+v^{*}\right)\left(\psi_{2}^{k+1}\right)^{\prime}\right](c)-A^{\prime} \psi_{2}^{k+1}(c) .
$$


Existence and uniqueness of solution of problems (58) and (59) can be proved using the same argument used for the demonstration of existence and uniqueness of the solution of the concentration equations in subdomains given in the following section.

\subsection{Existence and uniqueness of the solutions of the concentration equations on subdomain problems}

Let $1 \leq i \leq 5, a_{i}^{1}(v, \varphi)$ and $a_{i}^{2}(v, \varphi)$ be the followings bilinear forms :

$$
\begin{aligned}
& a_{i}^{1}(v, \varphi)=d_{i} \int_{0}^{c} v^{\prime}(x) \varphi^{\prime}(x) d x+\frac{1}{2} \int_{0}^{c}\left(b(x)+\frac{m_{i} V}{3 \delta}\right)\left(v(x)^{\prime} \varphi(x)-\varphi^{\prime}(x) v(x)\right) d x \\
& -\frac{1}{2} \int_{0}^{c} b^{\prime}(x) v(x) \varphi(x) d x-A v(c) \varphi(c)+\gamma_{i} v(0) \varphi(0)
\end{aligned}
$$

and

$$
\begin{aligned}
& a_{i}^{2}(v, \varphi)=d_{i} \int_{c}^{3 \delta} v^{\prime}(x) \varphi^{\prime}(x) d x+\frac{1}{2} \int_{c}^{3 \delta}\left(b(x)+\frac{m_{i} V}{3 \delta}\right)\left(v(x)^{\prime} \varphi(x)-\varphi^{\prime}(x) v(x)\right) d x \\
& -\frac{1}{2} \int_{c}^{3 \delta} b^{\prime}(x) v(x) \varphi(x) d x+B v(c) \varphi(c)
\end{aligned}
$$

for $(v, \varphi) \in H \times H$.

By multiplying the two systems (55) and (56) by $\varphi \in H, H$ is from (21), we obtain for all $n \geq 0, k \geq 0$ :

$$
\begin{aligned}
& a_{i}^{2}(v, \varphi)=-m_{i} \int_{0}^{c}\left(v_{i, n}(x)+w_{i}^{*}\right)(\psi)^{\prime}(x) \varphi(x) d x+\int_{0}^{c} \widetilde{S}_{3}\left(v_{3, n}, v_{4, n}, v_{5, n}\right)(x) \varphi(x) d x \\
& -\left(\gamma_{i}+\frac{m_{i} V}{3 \delta}\right) w_{i}^{*} \varphi(0)-\frac{m_{i} V}{6 \delta} v_{i, n}(0) \varphi(0)+\left(\lambda_{i}^{k}+m_{i}\left(v_{i, n}(c)+w_{i}^{*}\right)(\psi)^{\prime}(c)\right) \varphi(c), \\
& a_{i}^{2}(v, \varphi)=-m_{i} \int_{c}^{3 \delta}\left(v_{i, n}(x)+w_{i}^{*}\right)(\psi)^{\prime}(x) \varphi(x) d x+\int_{c}^{3 \delta} \widetilde{S}_{3}\left(v_{3, n}, v_{4, n}, v_{5, n}\right)(x) \varphi(x) d x \\
& +(B-A) v_{i 1}^{k+1}(c) \varphi(c) \\
& -\left(d_{i}\left(v_{i 1}^{k+1}\right)^{\prime}(c)-\left(\frac{1}{2}\left(b(c)+\frac{m_{i} V}{3 \delta}\right)+A\right) v_{i 1}(c)+m_{i}\left(v_{i, n}(c)+w_{i}^{*}\right)(\psi)^{\prime}(c)\right) \varphi(c)
\end{aligned}
$$

and

$$
\lambda_{i}^{k+1}=d_{i}\left(v_{i 2}^{k+1}\right)^{\prime}(c)-\left(\frac{1}{2}\left(b(c)+\frac{m_{i} V}{3 \delta}\right)+A\right) v_{i 2}^{k+1}(c) .
$$


Definition 4 Let $n \geq 0, k \geq 0$ and $\left(v_{i, n}, v_{i 1}^{k}, \psi\right) \in\left(C^{2}([0, c])\right)^{3}$, we say that $v_{i 1}^{k+1}$ is a solution of (55) if and only if for all $\varphi \in H^{1}(0, c)$ :

$$
\left\{\begin{array}{l}
v_{i 1}^{k+1} \in H^{1}(0, c) \\
a_{i}^{1}\left(v_{i 1}^{k+1}, \varphi\right)=-m_{i} \int_{0}^{c}\left(v_{i, n}(x)+w_{i}^{*}\right)(\psi)^{\prime}(x) \varphi(x) d x \\
+\int_{0}^{c} \widetilde{S}_{3}\left(v_{3, n}, v_{4, n}, v_{5, n}\right)(x) \varphi(x) d x \\
-\left(\gamma_{i}+\frac{m_{i} V}{3 \delta}\right) w_{i}^{*} \varphi(0)+\frac{m_{i} V}{6 \delta} v_{i, n}(0) \varphi(0) \\
+\left(\lambda_{i}^{k}+m_{i}\left(v_{i 1}^{k}(c)+w_{i}^{*}\right)(\psi)^{\prime}(c)\right) \varphi(c)
\end{array}\right.
$$

Then we have the following result :

Proposition 5 Suppose that $A \leq 0$, then the problem (55) has a unique solution $v_{i 1}^{k+1} \in C^{2}([0, c])$.

Proof. Since $A \leq 0$ and $\gamma_{i} \geq 0$, it follows that the form $a_{i}^{1}$ is coercive. Then we proceed by the same manner as in the proof of proposition 2 to deduce the result.

Definition 6 Let $n \geq 0,\left(v_{i, n}, \psi\right) \in C^{2}([c, 3 \delta]) \times C^{2}([c, 3 \delta])$ and $v_{i 1}^{k+1}$ given by (66), we say that $v_{i 2}^{k+1}$ is a solution of (56) if and only if for all $\varphi \in H^{1}(c, 3 \delta)$ :

$$
\left\{\begin{array}{l}
v_{i 2}^{k+1} \in H^{1}(c, 3 \delta), \\
v_{i 2}^{k+1}(3 \delta)=0, \\
a_{i}^{2}\left(v_{i 2}^{k+1}, \varphi\right)=-m_{i} \int_{c}^{3 \delta}\left(v_{i, n}(x)+w_{i}^{*}\right)\left(\psi_{2}\right)^{\prime}(x) \varphi(x) d x \\
+\int_{c}^{3 \delta} \widetilde{S}_{3}\left(v_{3, n}, v_{4, n}, v_{5, n}\right)(x) \varphi(x) d x+(B-A) v_{i 1}^{k}(c) \varphi(c) \\
-\left(d_{i}\left(v_{i 1}^{k}\right)^{\prime}(c)-\left(\frac{1}{2}\left(b(c)+\frac{m_{i} V}{3 \delta}\right)+A\right) v_{i 1}^{k}(c)+m_{i}\left(v_{i, n}(c)+w_{i}^{*}\right)(\psi)^{\prime}(c)\right) \varphi(c),
\end{array}\right.
$$

Then we have the following result :

Proposition 7 Suppose that $B \geq 0$, then the problem (56) has a unique solution $v_{i 2}^{k+1} \in C^{2}([c, 3 \delta])$.

Proof. Since $B \geq 0$ it follows that the form $a_{i}^{2}$ is coercive. Then we proceed by the same manner as in the proof of proposition 2 to deduce the result. 


\subsubsection{Convergence of the subdomain decomposition algorithm}

To prove the convergence of the subdomain decomposition algorithm applied to problem (50), we consider two problems :

$$
\left\{\begin{array}{l}
L^{i} v_{i 1}=F_{i}\left(v_{1, n}, v_{2, n}, v_{3, n}, v_{4, n}, v_{5, n}, \psi\right) \quad \text { in }(0, c) \\
d_{i}\left(v_{i 1}\right)^{\prime}(c)-\left(\frac{1}{2} B_{0}(c)+A\right) v_{i 1}(c)= \\
d_{i}\left(v_{i, n}\right)^{\prime}(c)-\left(\frac{1}{2} B_{0}(c)+B\right) v_{i, n}(c) \\
G_{i}\left(v_{i 1}, \psi\right)(0)=-d_{i}\left(v_{i, n}\right)^{\prime}(0) .
\end{array}\right.
$$

then

$$
\left\{\begin{array}{l}
L^{i} v_{i 2}=F_{i}\left(v_{1, n}, v_{2, n}, v_{3, n}, v_{4, n}, v_{5, n}, \psi\right) \quad \text { in }(c, 3 \delta) \\
d_{i}\left(v_{i 2}\right)^{\prime}(c)-\left(\frac{1}{2} B_{0}(c)+B\right) v_{i 2}(c)= \\
d_{i}\left(v_{i 1}\right)^{\prime}(c)-\left(\frac{1}{2} B_{0}(c)+B\right) v_{i 1}(c), \\
v_{i 2}(3 \delta)=0,
\end{array}\right.
$$

Proposition 8 Suppose that c is near 0 or near $3 \delta$, the sequence $v_{i 1}^{k}$ converge to $v_{i 1}$ in $C(0, c)$ and he sequence $v_{i 2}^{k}$ converge to $v_{i 2}$ in $C(c, 3 \delta)$

Proof. Let us define the errors $e_{i j}^{k}=v_{i j}-v_{i j}^{k}, 1 \leq i \leq 5, j=1,2$, and study their behavior as $k$ grows.

We prove the following inequality :

$$
\left\|e_{i 1}^{k+1}\right\|_{\infty} \leq \gamma\left\|e_{i 1}^{k}\right\|_{\infty} \text { and }\left\|e_{i 2}^{k+1}\right\|_{\infty} \leq \gamma\left\|e_{i 2}^{k}\right\|_{\infty},
$$

where $\gamma<1$.

By proceeding straightforwardly, the following iterative scheme is obtained for the error functions :

$$
\begin{gathered}
e_{i j}^{0}=v_{i, n}, \\
\left\{\begin{array}{l}
\left.L^{i} e_{i 1}^{k+1}=0 \quad \text { in }\right] 0, c[ \\
d_{i}\left(e_{i 1}^{k+1}\right)^{\prime}(c)-\left(\frac{1}{2} B_{0}(c)+A\right) e_{i 1}^{k+1}(c)= \\
d_{i}\left(e_{i 2}^{k}\right)^{\prime}(c)-\left(\frac{1}{2} B_{0}(c)+B\right) e_{i 2}^{k}(c) \\
e_{i 1}^{k+1}(0)=0 .
\end{array}\right.
\end{gathered}
$$

and

$$
\left\{\begin{array}{l}
\left.L^{i} e_{i 2}^{k+1}=0 \quad \text { in }\right] c, 3 \delta[ \\
d_{i}\left(e_{i 2}^{k+1}\right)^{\prime}(c)-\left(\frac{1}{2} B_{0}(c)+B\right) e_{i 2}^{k+1}(c)= \\
d_{i}\left(e_{i 1}^{k+1}\right)^{\prime}(c)-\left(\frac{1}{2} B_{0}(c)+B\right) e_{i 1}^{k+1}(c), \\
e_{i 2}^{k+1}(3 \delta)=0 .
\end{array}\right.
$$


Setting :

$$
\begin{aligned}
& c_{1}=\frac{1}{d}\left(\frac{1}{2} B_{0}^{i}(c)+A\right), \\
& c_{2}=\frac{1}{d}\left(\frac{1}{2} B_{0}^{i}(c)+B\right), \\
& f(x)=\int_{c}^{x} \exp \left(\frac{B_{0}^{i}(t)}{d}\right) d t, x \in[0,3 \delta], \\
& G(x)=\int_{x}^{3 \delta} f(y) d y, x \in[c, 3 \delta], \\
& H(x)=\int_{0}^{x} f(y) d y, x \in[0, c] .
\end{aligned}
$$

The corresponding solutions are :

$$
e_{i 1}^{k+1}(x)=\frac{\frac{\left(1-c_{1} G(c)\right)}{H(c)}-c_{2}\left(1-c_{1} G(c)\right)}{\left(1-c_{1} H(c)\right)\left(1-c_{2} G(c)\right)} e_{i 1}^{k}(c) H(x)
$$

and

$$
e_{i 2}^{k+1}(x)=\frac{\left(1-c_{1} G(c)\right)\left(1-c_{2} H(c)\right)}{\left(1+c_{2} G(c)\right)\left(1-c_{1} H(c)\right)} e_{i 2}^{k}(c) G(x) .
$$

It follows that :

$$
\left\|e_{i 1}^{k+1}\right\|_{\infty} \leq \gamma_{1}\left\|e_{i 1}^{k}\right\|_{\infty} \text { and }\left\|e_{i 2}^{k+1}\right\|_{\infty} \leq \gamma_{2}\left\|e_{i 2}^{k}\right\|_{\infty},
$$

where

$$
\gamma_{1}=\left|\frac{\left(1-c_{1} G(c)\right)-c_{2}\left(1-c_{1} G(c)\right) H(c)}{\left(1-c_{1} H(c)\right)\left(1-c_{2} G(c)\right)}\right|
$$

and

$$
\gamma_{2}=\left|\frac{\left(1-c_{1} G(c)\right)\left(1-c_{2} H(c)\right)}{\left(1+c_{2} G(c)\right)\left(1-c_{1} H(c)\right)} G(c)\right| .
$$

By comparing $\left(\gamma_{j}\right)^{2}, j=1,2$ and 1 we deduce that :

$$
\gamma_{j}<1 \text { if and only if }(G(c)-F(c)) \Theta(A, B)<0,
$$

where

$$
\begin{aligned}
\Theta(A, B) & =\frac{B-A}{d}\left(2-\frac{1}{d}(F(c)+G(c))\left(B_{0}^{i}(c)+A+B\right)\right. \\
& \left.+\frac{2}{d} F(c) G(c)\left(\frac{B_{0}^{i}(c)}{2}+A\right)\left(\frac{B_{0}^{i}(c)}{2}+B\right)\right) .
\end{aligned}
$$


We have $c(c-3 \delta)$ is near 0 , then $\mathrm{F}(\mathrm{c}) \mathrm{G}(\mathrm{c})$ is near 0 , it follows that $\Theta(A, B)$ and $\frac{B-A}{d}\left(2-\frac{1}{d}(F(c)+G(c))\left(B_{0}^{i}(c)+A+B\right)\right)$ have the same sign. Then $\Theta(A, B)>0$, which implies that :

$$
(G(c)-F(c)) \Theta(A, B)<0 .
$$

Finally we take

$$
\gamma=\max \left(\gamma_{1}, \gamma_{2}\right)
$$

The convergence of the subdomain decomposition algorithm applied to the potential equation solution can be proved using the same argument.

\section{Numerical result}

The method developed in previous sections was applied to a real experimental case.

The parameter $\delta=1.134110^{-5}$ gives the domain $(0,3 \delta)$. The diffusion constant are the following $d_{1}=6.8 * 10^{-} 10 ; d_{2}=7.6 * 10^{-} 10 ; d_{3}=9.31 * 10^{-} 9$; $d_{4}=1.33 * 10^{-} 9 ; d_{5}=1.065 * 10^{-} 8$.

The charges are the followings $z_{1}=2 ; z_{2}=2 ; z_{3}=1 ; z_{4}=-2 ; z_{5}=-1$. The electrical mobility $m_{i}$ of species $A_{i}$ are given: $m_{1}=5.2133 * 10^{-} 8 ; m_{2}=$ $5.827 * 10^{-} 8 ; m_{3}=3.569 * 10^{-} 7 ; m_{4}=-5.179 * 10^{-} 8 ; m_{5}=-8.295 * 10^{-} 8$.

The fictitious boundary values of the solutions in $x=3 \delta$ are the followings $w_{1}(3 \delta)=500 ; w_{2}(3 \delta)(2)=10 ; w_{3}(3 \delta)=10 ; w_{4}(3 \delta)=514.9 ; w_{5}(3 \delta)=0.05$ and $\Phi(3 \delta)=0$. In $x=0, \Phi(0)=-0.85 * 10^{-} 2$.

In figure 1 we plot the concentration of $\mathrm{Ni}^{2+}$ obtained in the simulation. The concentration of $\mathrm{Fe}^{2+}$ is presented in figure 2 .

The domain decomposition considered is in two subdomains with a fictitious boundary in $c=\delta$. The parameters of the fictitious boundary conditions in $c$ are $A=0$ and $B=1.2 * 10^{-4}$. Linear problem in each subdomain is solved using a finite element method.

\section{Conclusion}

We present in this paper a numerical method to solve a systems of reaction diffusion equations coupled to a potential equation modeling an electrodeposition process. A subdomain decomposition technique taking account of the 


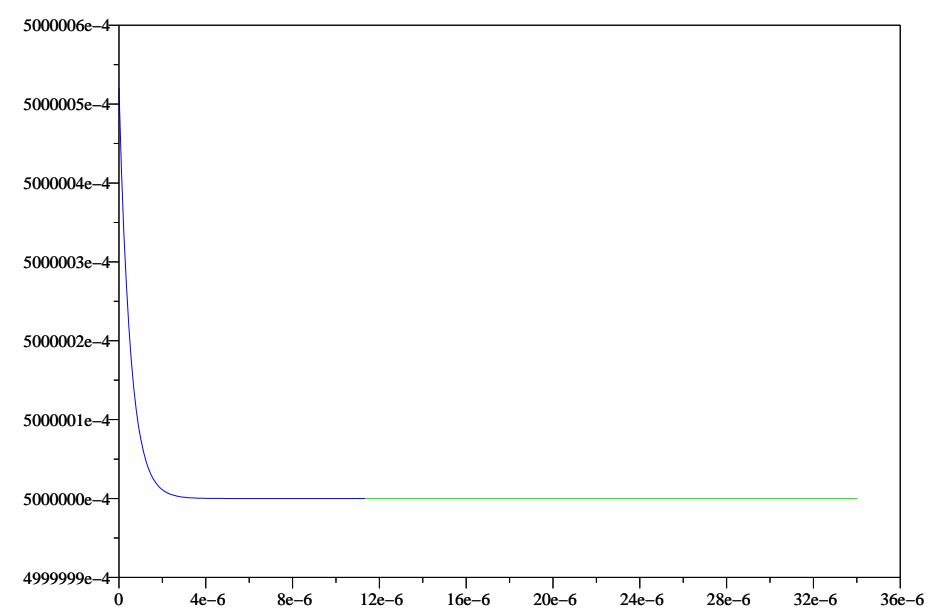

Figure 1: concentration of $N i^{2+}$

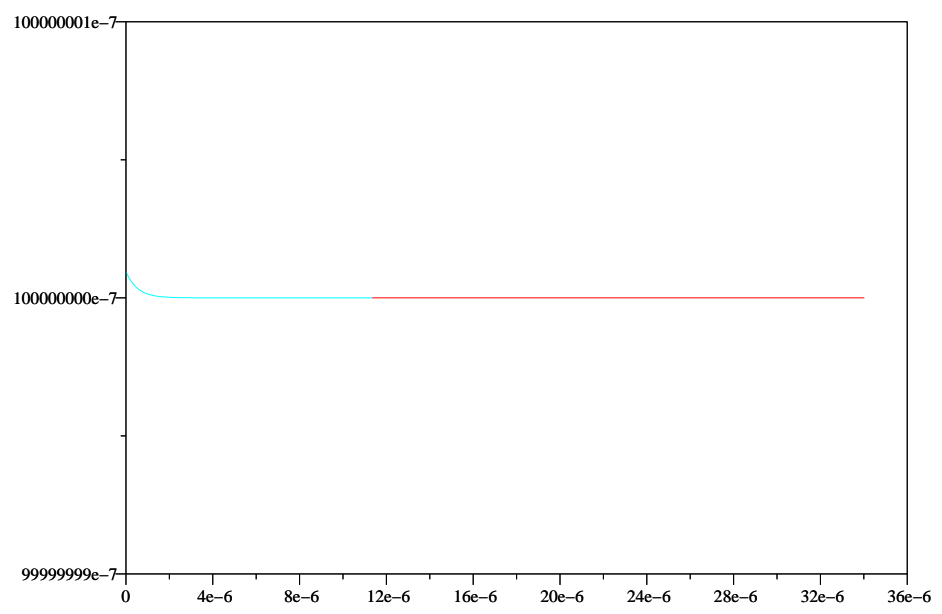

Figure 2: concentration of $\mathrm{Fe}^{2+}$

anisotropic behaviour of the solution is introduced. The proof of the convergence of the domain decomposition method is given. The numerical results 


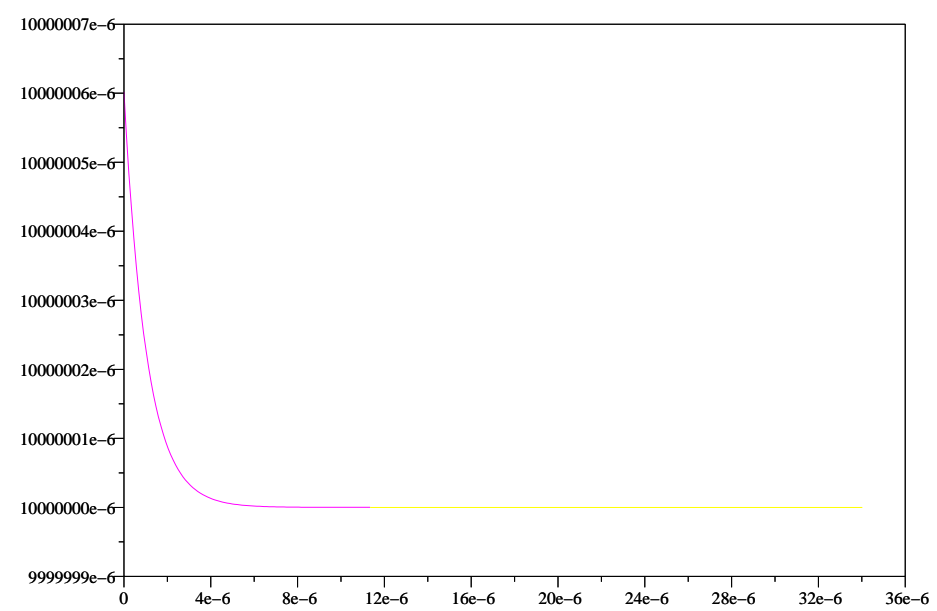

Figure 3: concentration of $H^{+}$

in a real case shows clearly that the process take place near the cathode.

\section{References}

[1] A. J. Bard and L. R. Faulkner, Electrochimie : Principes, méthodes et applications, Masson, Paris, (1983).

[2] J. O’M. Bockris, D. Drazic, and A. R. Despic, Electrochim. Acta, 4, 325, (1961).

[3] A. Brenner, Electrodeposition of alloys, Vol I, Academic Press, New York, (1963).

[4] D. Gangasingh and J. B. Talbot, J. Electrochem. Soc. 140, 669, (1993).

[5] F. Gastaldi, L. Gastaldi and A. Quarteroni, Adaptive domain decomposition methods for advection dominated equation, East-West J. Numer. Math, 4, 165-206, (1996).

[6] W.C. Grande and J. B. Talbot, J. Electrochem. Soc. 140, 675, (1993). 
[7] S. Hessami and C. W. Tobias, J. Electrochem. Soc. 136, 3611, (1989).

[8] J. Horkans, J. Electrochem. Soc. 126, 1861, (1979).

[9] T. Krause, L. Arulnayagam, and M. Pritzker, J. Electrochem. Soc. 144, 960, (1997).

[10] V. G. Levich, Physiochemical Hydrodynamics, Prentice-Hall, Englewood Cliffs, NJ, (1962).

[11] M. Matlosz, J. Electrochem. Soc. 140, 2272, (1993).

[12] J. Matulis and R. Slyzys, J. Electrochem. Soc. 9, 1177, (1964).

[13] A. Quarteroni and A. Valli, Domain decomposition methods for partial differential equations, Oxford, Clarendon, (1999).

[14] M. Ramasubramanian, S. N. Popova, B. N. Popova, R. E. White, and K.-M. Yin, J. Electrochem. Soc. 143, 2164, (1996).

[15] H. Schultz, and M. Pritzker, J. Electrochem. Soc. 145, 2033, (1998).

[16] C. W. Tobias, M. Eisenberg, and C .R. Wilke, J. Electrochem. Soc. 99, 359c, (1952).

[17] R. Y. Ying, P. K. Ng, Z. Mao, and R.E. White, J. Electrochem. Soc. 135, 2964, (1988). 